\title{
REDES DE CONTACTO SOCIALES. ¿FACTOR CLAVE PARA LA LABOR DE INVESTIGACION UNIVERSITARIA?
}

\author{
Yenia Melo Hermosilla* \\ Abelardo Castro Hidalgo ${ }^{* *}$
}

\begin{abstract}
RESUMEN: Dentro del ámbito de la labor universitaria, el nexo permanente entre docencia, investigación y difusión, constituye un factor de vital importancia. Bajo este prisma, un elemento fundamental para la formación permanente del personal académico es su compromiso con la realización de investigaciones, que contribuyan a generar nuevos conocimientos y a ampliar sus perspectivas de desarrollo. Siguiendo esta línea de razonamiento, este artículo examina el medio ambiente de investigación universitaria, específicamente, el tema de la vinculación o red de contactos de 19 investigadores, pertenecientes a diferentes áreas del conocimiento, con la sociedad circundante y el mundo de la investigación. Las bases teóricas del estudio se relacionan con dos niveles de análisis. El primero de naturaleza sociológica, se centra en la relación ciencia-sociedad. El segundo, de naturaleza psicológica, aborda aspectos que se refieren a la relación individuo-conocimiento. La perspectiva cualitativa, a través de una aproximación fenomenográfica, describe la metodología empleada en este estudio. Por ello, las percepciones del grupo de académicos participantes, fueron conocidas a través de entrevistas en profundidad. Los resultados revelan características de las redes de contacto, motivaciones que originan estos vínculos, estrategias relacionadas con su forma de construcción y de
\end{abstract}

* Profesora de Francés, Magister en Educación y alumna del programa de Doctorado en Educación (Facultad de Educación). Profesora asistente en Depto. de Curriculum e Instrucción, Universidad de Concepción.E-mail: ymelo@udec.cl

** Profesor de Filosofía, Doctor por la Universidad de Uppsala, Suecia y se desempeña como profesor titular del Departamento de Metodología de la Investigación e Informática de la Universidad de Concepción.E-mail: acastro@udec.cl 
utilización, efectos en la labor de investigación. El estudio concluye con reflexiones finales, entre las que se destacan posibles proyectos para futuras investigaciones sobre el tema abordado.

Palabras claves: Educación superior. Ambientes de investigación. Redes de contacto. Vinculación. Producción científica.

\section{DYNAMICS OF SOCIAL SCIENTIFIC CONTACT NETWORKS: A QUALITATIVE STUDY OF THEIR IMPACT ON THE RESEARCH WORK AT UNIVERSITIES}

ABSTRACT: As regards to university activity, the permanent link among teaching, research and dissemination plays a very important, vital role. Within such context, a fundamental element for the permanent training of the academic staff is their commitment to do research that contribute to generating new knowledge and to improving perspectives for development. Following this reasoning line, this qualitative study looks into the university research environment, and more specifically into the issue of creating links or networks among 19 researchers selected in different disciplines, and the surrounding community and research world. The theoretical framework includes two levels of analysis. The first one presents a sociological view on the relationship between society and science. The second one refers to the underlying psychological aspects in the relationship between individuals and knowledge. Through a phenomenographical approach, its methodology is based on the qualitative tradition. The researchers' own perceptions of their activities surfaced from in-depth interviews. Our results outlined the networks features, the driving forces in their development, their consolidation mechanisms and the way contacts are used and how they influence research. As a conclusion, this study brings forward some final reflections, among which are possible projects for future research.

Key words: Higher education. Research environments. Networks. Scientific production.

\section{Presentación}

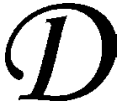

urante los últimos años, la educación superior ha ido experimentando cambios significativos y se ha visto enfrentada a nuevos desafíos relacionados con la diversidad de escenarios donde debe desarrollarse. Al mismo tiempo, estos cambios y desafíos constituyen un tema que concita especial interés en diferentes esferas de la sociedad.

Actualmente, el mundo moderno requiere de una integración del conocimiento en un proceso multidisciplinario, que incluye el 
quehacer científico, tecnológico, social y cultural, con el fin de ampliar sus perspectivas de desarrollo.

Dentro de este contexto, las universidades del nuevo milenio, con el fin de mantener un equilibrio entre calidad, eficiencia y equidad, no podrán permanecer aisladas, sino que, con una visión de futuro, propiciarán acciones tendientes a enriquecer sus contactos con otros sectores del entorno social, asociados al mundo de la cultura y de las artes, al sector laboral y productivo, a sectores relacionados con educación y salud etc. Además de potenciar los contactos antes mencionados, estas instituciones deberán responder a demandas vinculadas con una administración eficiente y con la búsqueda de fuentes alternativas de financiamiento (Cassier, 1998; Soria, 1993).

\section{El problema}

Una de las misiones esenciales de la Universidad es desempeñar un rol protagónico en el ámbito de las ciencias, las artes y las letras. Dentro de esta perspectiva, la investigación constituye una labor de vital importancia y se concibe en estrecha relación con la docencia y la difusión. Sin embargo, en contraste con las afirmaciones anteriores, nos vemos enfrentados a una realidad que genera preocupación en diferentes sectores de nuestra sociedad.

Antecedentes proporcionados por distintas fuentes indican que en nuestro país existe un aislamiento de la ciencia y se requiere proyectar acciones que estimulen, aún más, el intercambio, la cooperación internacional (Conicyt, 1993).

Por estos motivos, en un mundo en que la información y el conocimiento adquieren dimensiones cada vez mayores, incluso insospechadas, se requiere aumentar la cantidad de recursos humanos para la investigación y existe consenso en que se hace urgente potenciar el sistema nacional de investigadores, en las distintas ramas del saber.

Acuerdos del último claustro académico, realizado en esta Universidad, indican que se requiere de una política nacional de investigación más clara en lo que respecta a sus objetivos, áreas prioritarias y mecanismos de evaluación (Documento de Claustro Académico U. de C., 1998).

Dentro de esta perspectiva, resulta interesante fortalecer programas institucionales que generen y consoliden grupos de 
investigación uni y multidisciplinarios, con el objeto de favorecer el avance del conocimiento en el área de la Ciencia y la Tecnología, así como en las áreas de Educación, Ciencias Sociales y Humanidades. Al mismo tiempo, se hace absolutamente imprescindible potenciar al investigador, en particular, a fin de incrementar su capacidad científica.

Desde esta óptica, se hace urgente conocer aspectos relacionados con el medio ambiente de investigación, entre otros, la dinámica de las redes de contacto científico sociales y su incidencia en la labor de investigación. De esta forma, al reflexionar sobre estas realidades, surgió nuestro problema: En el contexto del medio ambiente de investigación ¿Cuál es la dinámica de la lógica interna de funcionamiento de las redes de contacto y cómo ellas influyen en la labor de investigación del profesor universitario?

\section{Aspectos teóricos}

En relación al marco teórico de esta investigación, éste contempla dos niveles de análisis. El primero de ellos, presenta una visión sociológica acerca de la relación entre ciencia y sociedad. El segundo nivel, aborda aspectos psicológicos referidos a la relación del individuo con el conocimiento.

\section{Relación ciencia-sociedad}

El punto central de la mayor parte de los estudios asociados a este tema ha sido el problema de "dependencia o libertad de la investigación respecto de la sociedad”. Específicamente, en lo que concierne a valores generales, a la organización y a las tendencias de la investigación científica, así como a la influencia que ésta pueda ejercer en la sociedad.

Dentro de esta perspectiva, existe un flujo continuo de escritores que han reflexionado sobre este tema y tienen distintas concepciones como puntos de partida (Gustavsson, 1971). Algunos de ellos se refieren a la existencia de lazos entre ciencia y sociedad, y sus opiniones concuerdan al atribuir un carácter evolutivo al conjunto de conocimientos acumulados por el hombre en íntima relación con el contexto histórico. Escritos realizados por filósofos, historiadores y sociólogos de diferentes épocas lo han ido demostrando en forma progresiva (Vinck, 1995). 
En el marco de las ideas anteriormente analizadas, el filósofo francés Bruno Latour (1995) afirma que existen estrechas conexiones entre el trabajo de investigación científica, sus resultados y el medio social que lo rodea. En este contexto, se observa la existencia de dos mitos referidos a la actividad científica. El primero se basa en la necesidad de proteger la investigación de ideologías e intereses externos. El segundo mito, por el contrario, se origina al exterior del mundo científico y tiende a proteger a la sociedad de la ciencia y sus avances, que en ciertas ocasiones podrían constituir una amenaza para la sociedad.

Tras reflexionar sobre los planteamientos anteriores, resulta difícil demostrar tan fuertes lazos entre el conocimiento y el contexto social, quizás tan difícil como conseguir pruebas científicas de la "total" libertad de la ciencia. En efecto, en lo que respecta a la ciencia moderna, las cosas parecieran ser distintas y resulta interesante revisar planteamientos distintos de los anteriores.

En lo que concierne a la concepción intrínseca de la ciencia, otros escritores y estudiosos del tema señalan que ésta tiene su propia fuerza de desarrollo y es independiente de los grupos externos, incluyendo los valores dominantes y las formas de organización de la sociedad (Vinck, 1995).

Esta posición considera que las actividades científicas han sido desarrolladas en ambientes e instituciones que han gozado de un alto grado de autonomía y no han sido presionadas para obtener beneficios desde el punto de vista económico. En este sentido, el desarrollo de la ciencia se concibe como producto del esfuerzo de un grupo de personas solas y tenaces, quienes, a pesar de las vicisitudes que se presentan, intentan demostrar sus hipótesis acerca de un fenómeno particular.

Siguiendo esta línea de pensamiento, pero enfocando el tema con matices diferentes, el sociólogo americano Robert Merton (1973) defiende la autonomía y la universalidad de la ciencia. Este autor atribuye a la ciencia un rol de institución social, con mecanismos de regulación y normas particulares, que le asignan un status distinto y autónomo. Además de favorecer el avance de las ciencias, estas normas lo protegen de ideología e intereses provenientes de los distintos sectores que componen la sociedad (Vinck, 1995).

Un análisis más profundo de esta discusión nos conduce a diferenciar dos aspectos fundamentales de la ciencia que son los siguientes: 
a) El proceso de investigación propiamente tal, mediante el cual se genera el conocimiento.

b) El resultado de ese proceso manifestado en un conjunto de conocimientos adquiridos, traducidos, entre otros, en textos conocimientos científicos expuestos en bibliotecas y revistas especializadas.

Por otra parte, no es posible concebir a la sociedad en términos de homogeneidad, en ella concurren distintos intereses y percepciones respecto del valor intrínseco de un determinado tipo de conocimiento y de su utilización.

Esta forma de enfocar el problema y la interacción de los elementos antes mencionados, nos conduce a pensar en momentos y grados de libertad de la ciencia en relación con un entorno social que no es homogéneo. También se podría afirmar que existen momentos o espacios de independencia y de dependencia de la ciencia, respecto del contexto social en que se encuentra inmersa, que se manifiestan de distinta manera de acuerdo al aspecto de la ciencia de que se trate (Castro 1992).

En lo que concierne al primer componente de la ciencia, proceso mediante el cual se genera el conocimiento, surge un momento en que la actividad es mucho más independiente. Este es el espacio en que el investigador se encuentra solo con sus ideas y las confronta a sus experiencias o conocimientos previos. Entonces la influencia social externa se reduce al mínimo. Es precisamente en este espacio en que el investigador, sólo con sus pensamientos, experimenta curiosidad por lo que sería deseable investigar. Dentro de este contexto, la ciencia concebida como proceso, adquiere un carácter más independiente, aunque no absoluto. Sin embargo, esta independencia se va perdiendo paulatinamente, en el momento en que el individuo comienza a formular su problema y a reflexionar acerca de los métodos más adecuados para abordarlo (Barnes, 1981). A medida que comienza a visualizar factores que podrían obstaculizar su investigación, va perdiendo, paulatinamente, la independencia inicial. Menos independiente es aún, cuando percibe que desarrolla un proyecto considerado inadecuado desde el punto de vista de la pertinencia o relevancia científica del mismo, o que se supone constituye una amenaza para ciertos sectores privilegiados de la sociedad.

En relación al segundo componente, el conjunto de conocimientos acumulados, es posible observar que cuando éste deja su condición 
de resultado científico puro, pasivo y se transforma en conocimiento científico activo, es decir, resulta en una aplicación dada, existen mayores posibilidades que sus líneas directrices sean influenciadas por los distintos sectores que conforman nuestra sociedad, de acuerdo a sus intereses.

En síntesis, según esta forma de enfocar el problema, se podría afirmar que existe un momento en que la ciencia como conocimiento acumulado es independiente. Esto sucede cuando nuevos conocimientos son "almacenados" sobre los ya existentes. La independencia se va perdiendo, gradualmente, a medida que se rompe el aislamiento de los conocimientos, produciéndose, de esta manera, su integración creciente y sus efectos en diferentes sectores del medio social.

Tras este análisis, es posible plantear que, de la misma forma en que se producen ciertos momentos de independencia o libertad de la ciencia, respecto de la sociedad en que el investigador se encuentra inmerso, se producen espacios de independencia y dependencia en el momento en que el individuo genera nuevos conocimientos. $\mathrm{Su}$ actividad, enfocada desde esta perspectiva, es un oscilar entre lo social e individual propio, sin que este último aspecto sea puro.

La idea de la existencia de un oscilar del científico entre los momentos de libertad en la creación científica y la existencia de momentos sociales de la misma, encuentra hoy apoyo en la corriente psicológica denominada constructivismo.

\section{Conocimiento desde el punto de vista cognitivo}

La literatura acerca de esta temática muestra que el aprendizaje y la obtención de conocimientos constituyen un fenómeno central en la vida individual y social de todos los seres humanos. Dentro de este contexto, otro aspecto del marco teórico de este estudio, se centra en el análisis de la línea de pensamiento cognoscitiva y en el fenómeno de la comunicación, éste último considerado como una función social del hombre.

En el marco de estas ideas, es preciso recordar que las bases y reflexiones filosóficas de la línea de pensamiento cognoscitiva se remontan al pensamiento griego. En efecto, ya en la Grecia Antigua se debatían temas y conceptos que, siglos más tarde, se transformarían en el núcleo de interés de una disciplina llamada psicología Cognitiva.

Posteriormente, esta forma de razonar resurge en la época medioeval, particularmente en las tendencias de modernismo, que 
propiciaban una mayor participación del hombre respecto de su medio y que cobran vigencia a través de los planteamientos constructivistas (Watzalawick, 1990).

Más tarde, este pensamiento es retomado por las ideas de la tradición filosófica occidental, a través del pensamiento racionalista e idealista de descartes, Leibnitz y Kant. Finalmente, resurge a través del movimiento cognitivista acual, representado por Piaget, Ausube, Vigotsky, entre otros.

Dentro de esta perspectiva, el supuesto que subyace a los planteamientos antes mencionados, dice relación con el desarrollo de estrategias, de la creatividad, del trabajo cooperativo, de capacidades metacognitivas, entre otras competencias (Barrón, 1991; Arancibia, 1990). El énfasis de estos factores contribuiría a propiciar la labor de investigación universitaria.

En esta óptica, resulta interesante revisar los planteamientos de investigador Jean Pierre Astolfi (1992), quien, en uno de sus escritos, aborda la relación entre el individuo y el conocimiento.

Este autor atribuye a los conceptos de información, conocimiento y saber, connotaciones especiales. En este sentido, la "información" tiene un carácter pasivo y considerada externa al individuo que la utiliza. Cuando se almacena, ya sea bajo la forma de código genético, revistas, cassettes, cartas perforadas, es para más tarde circular en el tiempo y en el espacio. Por el contrario, el "conocimiento" es subjetivo, inherente al individuo y a su historia. (Astolfi, 1992; Ausubel, 1983). A diferencia del concepto anterior, el conocimiento es construido por el propio sujeto y no puede ser transmitido. Esto significa que nos es imposible saber lo que una persona conoce en su globalidad, solo se puede inferir de manera parcial.

En contraste con los dos conceptos anteriores, el "saber" es construido por el propio sujeto, quien, a través de una formalización teórica y un lenguaje apropiado, logra expresar sólo una parte de su conocimiento. Al mismo tiempo, consiste en un verdadero esfuerzo de construcción intelectual, por parte del individuo, para hacer objetivo su conocimiento sobre algún aspecto en particular.

Siguiendo esta línea de pensamiento, en el momento en que se interpreta la realidad de una forma diferente, y luego, se expresa de manera formal ese pensamiento, se produce el saber y la generación de nuevas ideas. En este contexto, resulta interesante observar que hay un proceso cíclico, implícito en los tres conceptos antes mencionados: 
información, conocimiento, saber. Pareciera que se les atribuye un carácter de metamorfosis que les permite transformarse sucesivamente, obedeciendo a una cierta secuencia lógica.

Continuando con nuestro análisis, en el marco de las ideas presentadas, resulta de interés profundizar en el tema del conocimiento metacognitivo, éste último, referido a una "toma de conciencia respecto de las capacidades y limitaciones del pensamiento humano" (Arancibia, 1990; Rivière, 1988). El conocimiento metacognitivo está ligado a un proceso individual, y para efectos de esta investigación, se refleja en una toma de conciencia por parte del investigador acerca de cómo surgen nuevos temas de estudio, así como de las estrategias necesarias para el avance de su red de contactos y, por ende, de su labor de investigación.

De acuerdo a esta forma de razonar, el proceso de metacognición destaca al individuo como punto principal. Sin embargo, hoy, en esta nueva sociedad del conocimiento en la que estamos inmersos, se requiere más que eso. En efecto, se hace indispensable la construcción de nuevos saberes, como producto de interacción, tal como lo plantea el Construccionismo. Para que esto sea posible, se requiere de un soporte cognitivo, que se extiende más allá de los procesos metacognitivos que se mantienen en un nivel individual. Este soporte debiera ser una capacidad supracognitiva (Castro, 1999) que le permita al investigador, no solo ubicar su propia capacidad frente a la resolución de un problema dado sino, también, contar con las estructuras teóricas de otros investigadores, de áreas afines o convergentes y haciendo uso esas perspectivas para abordar problemas que le son propios.

Para complementar este análisis, resulta de interés revisar los planteamientos del psicólogo ruso Lev Vigotsky (1934-1960), quien postula que el cambio cognitivo es una actividad intelectual que permite generar nuevos conocimientos y se manifiesta en dos planos, primero social y, luego, psicológico.

En el plano social, el desarrollo del conocimiento se traduce en una actividad compartida o de interacción entre individuos. De esta forma, se establece un terreno común o "zona de construcción de conocimientos" (Newman, Griffin, Cole, 1998).

Es así como en el proceso de investigación, en el momento en que se genera una nueva idea y cuando ésta se desarrolla y madura, es de vital importancia el contacto con otros investigadores que permitan superar el punto de vista propio y coordinar opiniones con 
el pensamiento de otras personas que pueden encontrarse interesadas en la temática de investigación.

Tras las reflexiones anteriores, se hace evidente la necesidad de estudiar con mayor precisión el cómo se manifiestan las relaciones sociales científicas, el sentido que los investigadores dan a ésta y, finalmente, el cómo el investigador "usa" su red social concebida en un aspecto social amplio y en otro, restringido al ámbito científico.

\section{Metodología de la investigación}

La investigación se abordó desde una perspectiva cualitativa, mediante una aproximación fenomenográfica. Esta última considera las diferentes formas de los académicos de percibir, entender y conceptualizar un determinado fenómeno en estudio (Marton, 1978). Dentro de esta perspectiva, los docentes fueron invitados a reflexionar acerca de su propia experiencia en el campo de la investigación.

La muestra, seleccionada durante el año 1998, comprende a un grupo de 19 académicos, pertenecientes a las áreas de Cs. Naturales, Cs. Sociales y Tecnología. Saturación y amplitud describen el criterio utilizado para su selección. En efecto, se procedió a seleccionar a un grupo heterogéneo de personas que hubiesen participado en proyectos de investigación en el transcurso de los últimos 3 años y que tuvieran actividades de docencia en la Universidad. Luego, estas personas fueron entrevistadas en profundidad, hasta que sus discursos comenzaron a repetirse y no evidenciaron nada nuevo (Bisquerra, 1989; Taylor y Bodgan, 1986).

Cabe señalar que la pauta de entrevista fue objeto de una validación semántica, con el fin de asegurar la claridad de las preguntas y su coherencia con los objetivos del estudio. Además, se informó con anterioridad a los entrevistados sobre el objetivo del tema en estudio, con el propósito de establecer un clima de confianza, indispensable para un trabajo de esta naturaleza. Al ser llevadas a cabo en la muestra seleccionada para la investigación, las entrevistas fueron grabadas, tras garantizar el anonimato de los participantes.

Posteriormente, se procedió a transcribir las respuestas y a establecer categorías, consignando en cada una de ellas testimonios de los investigadores, que permitieran atribuir un significado a la información obtenida.

$\mathrm{Al}$ efectuar el análisis, las preguntas se dividieron en tres secciones, de acuerdo a las áreas temáticas abarcadas: antecedentes generales de los 
investigadores participantes, aspectos relacionados con el área de investigación y temas relacionados con las redes de contacto entre académicos y con el resto de la sociedad.

\section{Resultados}

Los resultados de esta investigación nos permitieron apreciar que indican que la existencia de puntos de encuentro y de divergencia entre los académicos, al abordar los diferentes temas planteados en la entrevista. Dentro de este contexto, la diversidad fue un término recurrente a través de todo el estudio. En efecto, la pluralidad se reflejó en antecedentes generales de los investigadores, tales como título profesional, área del conocimiento que representan, instituciones en que realizaron estudios de Postgrado e, incluso, en el plano de la experiencia y años de servicio en la Universidad. En cuanto a los temas abordados en las investigaciones, observamos que éstos se diversificaban en tres áreas de mayor amplitud: Cs. Naturales, Cs. Sociales y Tecnología. Sin pretender generalizar, resulta interesante observar que la diversidad antes mencionada se manifiesta además en la pluralidad de líneas de investigación existentes en la Universidad.

No obstante, los resultados antes mencionados revelan que los aspectos estudiados pueden ejercer un efecto significativo en el ámbito de la gestión, relacionada con el cómo potenciar la labor que realiza el investigador en la Universidad.

En definitiva, el conjunto de opiniones nos permitió responder a las interrogantes planteadas inicialmente.

\section{Construcción de una red social amplia y su significado para determinar la posibilidad y ejecución de la investigación propia}

En cuanto a los factores que motivan al investigador a construir una red de vínculos y al significado que les asignan...

1.1. El nivel de pertinencia de sus estudios, en un contexto social, fuera de la universidad

Los investigadores manifiestan interés por conocer otras realidades que les permitan enriquecer el campo de conocimiento de sus respectivas áreas de trabajo, en el ámbito social, de la salud, cultural 
y ecológico. Por cierto, son de vital importancia y contribuyen a concretar y a desarrollar proyectos e investigaciones que tengan impacto en la sociedad en la que se encuentran inmersos. Por estas razones, señalan:

Ahora, es un tema que se trata de estudiar internacionalmente porque hay una preocupación mundial por el tema de los pobres. El tema de mi última investigación surge de la necesidad de estudiar los problemas que tiene una sociedad que crece, que tiene éxito económico, pero que deja al margen de ese éxito, de ese progreso, a un sector importante de la sociedad.

Desde el punto de vista científico, son moléculas de tanto interés... aportar nuevas moléculas y aumentar la potencialidad de los tests biológicos que tenemos, para encontrar nuevos fármacos. De esta manera, se han encontrado aproximadamente el 59\% de los fármacos que se usan en el mundo... obviamente tienen que tener uso en la vida de la gente... Ese es el espíritu de estos proyectos.

Hay aspectos de la historia local que no están registrados y es importante que se conozcan y sean reconocidos en toda su magnitud... Esta ciudad tiene una especie de nostalgia por su historia...

\subsection{Las posibilidades de apoyo financiero, indispensables para el desarrollo de proyectos de investigación}

Los académicos señalan que los aportes de esta naturaleza son indispensables para desarrollar los proyectos de investigación, acceder a mayores recursos tecnológicos, invitar a profesores visitantes, integrar a co investigadores, financiar viajes y estadías. Al mismo tiempo, esto permite a los investigadores concretar proyectos de vida propios que constituyen un estímulo personal, un premio a sus méritos en investigación. En este contexto, los docentes expresan:

Yo creo que uno no tiene que sentarse en su escritorio a esperar que le vengan a entregar recursos... es uno el que tiene que salir a buscar los fondos... y como es competitivo, no es fácil, y hay que ser perseverante...

Hay que detectar a la gente que va a tomar las decisiones, del sector privado y hacerlos partícipes con antelación de la idea, porque si la conocen recién, en un documento que les llega a sus manos, no alcanzan a madurar las ideas...

El intercambio te abre los ojos a las posibilidades de financiamiento y eso es fundamental... Una característica de los investigadores que tienen fondos 
en esta universidad es la capacidad para detectar las necesidades del ambiente...

\section{La red social científica como base para conocer la relevancia científica "pura"}

Respecto de la forma cómo se consolidan y se usan las redes de contacto, observamos que ello ocurre a través del intercambio de ideas con colegas de la Universidad y de otras Universidades, nacionales y extranjeras; la visita a centros de investigación, la participación y presentación de trabajos en congresos y simposios, tanto nacionales como internacionales. Observamos que los contactos antes mencionados constituyen un aporte real para coordinar convenios, concretar proyectos y generar trabajos en conjunto. De acuerdo a las opiniones de los académicos, se observan dos categorías de percepción:

2.1. La asistencia a congresos y el contacto permanente con otros investigadores es una oportunidad para detectar temas nuevos que pueden ser investigados y adaptados a la realidad chilena

En relación con la primera tendencia, el contacto con otros investigadores a través de congresos permite enriquecer las temáticas de investigación y traer nuevos enfoques que puedan tener proyecciones en nuestro país y plasmarse en futuras investigaciones, adaptándose a la realidad nacional y respetando sus características. Los académicos señalan:

Cuando voy a los congresos en países ya más avanzados, encuentro proyectos que para Chile pueden ser innovativos, pero otros ya los aplicaron. Todo eso implica un proceso muy largo... para adaptar esas ideas, no se trata de copiar...

En los congresos obviamente uno conoce gente y empieza a informarse de aspectos bien interesantes que puede eventualmente ir incorporando y dar origen a nuevas investigaciones. En ese sentido mi visita a España ha sido muy fructífera para poder visualizar a futuro algunas cosas complementarias a las que estoy haciendo.

De hecho las investigaciones con gente de Canadá surgieron de un Seminario realizado en ese país sobre otro tema. Entonces, ahí se gestó un Proyecto de Desarrollo Comunitario. Yo creo que lo eventos nacionales e internacionales son siempre un espacio para hacer surgir nuevas iniciativas. 
2.2. La asistencia a congresos y el contacto con otros investigadores permiten al investigador revisar su temática de investigación, comparándola con la de otros investigadores que tienen una linea de investigación similar $y$, de esta forma, constatar la vigencia del tema

En lo que concierne a la segunda tendencia de opinión, la asistencia a congresos y el contacto con otros investigadores permiten reflexionar, discutir e intercambiar ideas sobre diversos aspectos relacionados con las investigaciones. De esta forma, el investigador realiza una análisis introspectivo que le permite determinar su nivel de conocimientos y avances en su respectiva línea de investigación y comparase con el nivel de avance de otros académicos que se interesan en la misma área temática. Los académicos manifiestan:

Yo diría que la asistencia a congresos es la principal fuente de inspiración en lo que son las líneas de investigación porque ahí uno conoce cuál es el estado del arte y uno se mantiene más fácilmente en la frontera del conocimiento de lo que uno está haciendo.

Nosotros tenemos una norma aquí en el laboratorio, cada vez que uno sale al extranjero, medimos nuestro éxito de la salida en el impacto que va a tener tanto en nuestro trabajo de investigación. Como en ideas, tengo que serle franco...

Cuando se asiste a congresos extranjeros, hay especialistas que acuden allí... con ellos se conversa... hay un intercambio de ideas... Puede que nosotros entreguemos ideas, pero también recogemos ideas...

\section{Construcción del conocimiento propio}

En el transcurso de las entrevistas se vislumbra el sentido que adquieren las redes en cuanto al trabajo propio del investigador. En lo esencial, la red es un punto de referencia donde el investigador prueba una idea, capta tendencias que pueden serle útiles para concretar y desarrollar nuevos proyectos, detecta ideas de otros investigadores que pueden ser de utilidad para sus propios proyectos y se enriquece con los aportes intelectuales de otros personas.

Esto nos indica que estas redes permiten confrontar experiencias análogas y enfocar problemas desde distintas perspectivas, incorporando las opiniones de investigadores de distintos puntos del planeta y el aporte de otras personas, que pueden encontrarse dentro o fuera del ámbito de la Universidad. Bajo este prisma, apreciamos cuatro formas de utilización de las redes de contacto: 
3.1. El uso de las redes de contacto para ubicar el "eslabón perdido" o los conocimientos que faltan, obtener ayuda para estructurar el conocimiento propio, captar formas de aproximación y razonamiento respecto de un problema

En relación con esta primera forma de utilizar las redes de contacto, nos parece importante destacar que la vía de contacto personal adquiere un especial énfasis. En efecto, el conjunto de opiniones considera relevante el factor confianza que debe existir, necesariamente, al conformar un equipo de trabajo. En este sentido, al conformar estos vínculos, las redes electrónicas, como Internet, constituyen una ayuda, al permitir comunicarse, pero son impersonales y se requiere complementarlas con un contacto personal, si realmente se desea consolidar verdaderas redes de apoyo.

Desde esta perspectiva se enfatiza la discusión con alumnos de Pre y Postgrado y su integración a proyectos de investigación. Los académicos opinan:

Nosotros privilegiamos la colaboración internacional, ya sea con Universidades o Centros de investigación externos. El contacto personal es relevante, quita tiempo, pero obviamente, una conversación de 5 a 10 minutos puede ser la base de desarrollo de un proyecto importante.

El contacto personal con gente que te pueda ayudar, que tenga más experiencia o experiencias complementarias, yo creo que es vital. El internet permite comunicarse, pero es impersonal. Si tú no tienes el contacto mano a mano... uno a uno con la persona, va a ser difícil...

Bueno, la vía importante creo que es trabajar en grupos con otros colegas, discutir... Yo creo que la investigación no puede hacerla una persona aislada, tiene ayudantes, tiene colegas... Yo creo que un investigador que no incorpora a alumnos no tiene mucha proyección ni mucho éxito... ¡ $\mathrm{Si}$ los alumnos son la materia prima que genera toda esta investigación!

3.2. El uso de las redes de contacto para determinar como están abordando un problema, cuales son las metodologias empleadas, los aspectos teóricos, los posibles resultados y las proyecciones del estudio

En relación con esta segunda forma de usar las redes de apoyo, observamos que permite reorientar la investigación propia, hacerla mas coherente, conocer otros puntos de vista acerca de la presentación de trabajos y ampliar la gama de proyecciones de los estudios. Los entrevistados señalan: 
Se conversa sobre aspectos metodológicos, coherencia, por supuesto... yo diría líneas que surgen... El contacto más importante que yo encuentro y donde realmente uno necesita mucha interacción con gente que tenga más experiencia o experiencia en otra área, es en el momento de poder definir qué voy a hacer con lo que he logrado detectar. ¿Es realmente un problema o es algo que está resuelto y, por ignorancia, no sé que está resuelto? Esas son las interrogantes que uno trata de resolver y son la base del problema de la investigación en Chile.

He conversado con colegas de departamento para conocer sus puntos de vista sobre la presentación de trabajos... He tenido relación con gente de Alemania... yo envío una publicación y ellos me colaboran revisándola, dándome su opinión... Sin esa colaboración, es posible que los trabajos estuvieran allí, los informes, sin más discusión...

\subsection{El uso de las redes de contacto para captar la estructura intelectual $y$ las ideas de otros investigadores que enfocan el problema desde una perspectiva distinta}

Con este propósito, el factor confianza se genera del contacto en situaciones más informales, menos estructuradas, fuera del ámbito académico, como por ejemplo, el compartir a la salida de una conferencia, en un ambiente de común acuerdo. Los investigadores señalan:

Yo creo que es más importante en los congresos la conversación de pasillo... que lo que se necesita saber a través de la charla... sobre todo a nivel internacional. Si Ud. va a un congreso y se reúne con los especialistas "top" del tema, ya sean nacionales o internacionales... mi consejo es... atrápenlo a la salida y... conversen en otro lado...

En los congresos a que asisto, uno no va simplemente a escuchar a un señor dictar una conferencia... Acabo de estar en un congreso en EeUU, en Oregon, y realmente allí no había nada nuevo... Pero si tuve dos contactos muy interesantes, uno de ellos, que está en Francia, nos va a permitir resolver un problema practico de un proyecto que tenemos ahora. La otra cosa interesante es un contacto que espero genere un proyecto Fontec, a corto plazo... Me abrió los ojos para captar otros aspectos que son interesantes...

3.4. El uso de las redes de apoyo para discutir sobre eventos, seminarios, nuevas tecnologias, contenido de cursos que se imparten en la Universidad, asi como de las estrategias tendientes a mejorar la labor de docencia

Los investigadores señalan: 
La investigación tiene una interacción importante con la docencia, son relaciones sinérgicas...

Intercambiamos con la mayor parte de los colegas del depto. Hacemos por lo menos una vez al año un balance de lo que son los contenidos, de las deficiencias de los diferentes cursos. Entonces hay una especie de intercambio colectivo que me parece indispensable.

\section{Características de las redes de contacto}

En lo que concierne a las características de las redes de contacto, observamos que éstas son personales y cambian según los intereses de los investigadores por sus áreas de trabajo.

Esto nos conduce a pensar en una característica de vital importancia: los vínculos son dinámicos, varían. Las opiniones de los investigadores nos permiten vislumbrar las características de las redes desde dos ángulos:

4.1. Las redes de contacto son profundas. En algunos casos, se mantienen por años y dan origen a proyectos en conjunto

En relación con la primera forma de concebir las redes, asociada con su carácter profundo, apreciamos que los contactos permanecen por años y se desarrolla un grado de confianza mutua indispensable para el trabajo de investigación. Cuando estas se consolidan, se incorpora a estudiantes y a profesionales jóvenes como co-investigadores. Los investigadores señalan:

En mi caso, yo tengo mas relaciones con gente del extranjero que del mismo departamento o de la universidad. Justamente volvemos ahora a establecer nuevos convenios con el mismo centro y con gente que conocí hace ya 22 años...

Bueno, estos proyectos que estamos realizando ahora se han conversado en años anteriores, han surgido de esos contactos personales que maduran y... la gente viene a trabajar acá y nos conocemos... porque lo importante es establecer una relación de amistad, conocerse como personas... saber que esa persona va a responder y que vamos a tener las condiciones para trabajar.

Tengo alumnos trabajando conmigo... Yo integro resultados de mis investigaciones en mis clases de Pre grado y los alumnos de Post grado, todos están trabajando en aspectos del Fondecyt que actualmente tengo... 
4.2. Las redes de contacto son cambiantes, fluctúan, aparecen y desaparecen, dependiendo de los temas abordados por los investigadores

En lo que se refiere a la segunda forma de caracterizar a las redes, centrada en su carácter variable, observamos que en algunas ocasiones estas son amplias debido a que incluyen contactos tanto sociales como académicos, nacionales e internacionales. Abarcan un espectro grande y se producen incluso por casualidad, fuera del horario de trabajo, en encuentros con amigos o colegas. Incluso, algunos contactos son difusos. El nexo se establece a la vez con colegas de facultad, de otras facultades, de otras Universidades, con personeros de gobierno que deciden sobre políticas sociales, culturales y de preservación del patrimonio ambiental.

Por el contrario, en otras ocasiones, estos contactos constituyen un núcleo pequeño que se traduce en vínculos aleatorios como "las conversaciones de pasillo", más bien fortuitas, sin embargo, algunas de ellas constituyen la base para la generación de algún proyecto importante. En relación con este tema, los investigadores opinan:

Tengo contacto con colegas de departamento, con colegas de otras facultades, con todo el mundo... con autoridades municipales, con profesores de universidades nacionales e internacionales... Personalmente, tengo una fuerte labor de gestión y utilizo esta capacidad para interactuar, pero hay otros investigadores que son más bien reacios al mundo fuera de la cápsula de la Universidad, que prefieren estar tranquilos en su laboratorio...

Nuestro quehacer está muy ligado al medio externo, ya sea al sector público, al gobierno regional, los gobiernos locales, las municipalidades $y$, también, tiene que ver con el sector privado, las empresas, en las cuáles hacemos investigación aplicada.

He visitado varios centros de investigación. Bueno... en Latinoamérica: Perú, Ecuador, Colombia... He estado en México, en República Dominicana... Además he visitado Sudáfrica, Francia, Dinamarca, Inglaterra, E.E.U.U...

\section{Ventajas de una red de contactos}

Respondiendo a otra de las interrogantes inicialmente planteadas, apreciamos que estos contactos producen variados efectos, que favorecen el desarrollo de la labor de investigación.

Dentro de este contexto, observamos que el diálogo con sectores intra y extra universitarios constituye un factor de éxito, tanto para la creación como para el desarrollo de proyectos de investigación. 
Otro de los aspectos que favorece la realización de estudios es la coordinación que se produce entre los sectores que investigan, los que deciden sobre políticas en diferentes ámbitos (cultura, salud, trabajo, tecnología) y los sectores que requieren de apoyo en los ámbitos antes mencionados. Este trabajo en red permite detectar posibilidades de apoyo financiero y obtener recursos, indispensables para el desarrollo de las investigaciones.

Otro efecto positivo es que permiten conocer la realidad de otros mundos universitarios y mantenerse en la frontera del conocimiento. Al mismo tiempo, este intercambio estimula la creatividad entre investigadores y, de esta forma, se generan nuevas temáticas de estudio, adaptadas a la realidad de nuestro país. Dentro de este contexto, los investigadores manifiestan:

Este intercambio es vital porque estimula dos aspectos centrales: por un lado, la creatividad y, por otro lado, ayuda a detectar posibilidades de financiamiento... y eso es fundamental.

Como todavía estamos en un nivel en que tenemos problemas ambientales no superados, investigadores de Europa encuentran cierta forma de poder investigar en nuestros problemas ambientales... Esto permite una simbiosis entre los adelantos que tienen en Europa y los problemas medio ambientales que tenemos en Latinoamérica.

El contacto permite ir definiendo mejor los problemas... En investigación básica ayuda a identificar y a resolver cuestiones genéricas... es ahí donde el contacto sirve...

El intercambio contribuye al desarrollo de las investigaciones... Estimula el trabajo en conjunto, en su contexto teórico, metodológico y operativo, ya que el tema ambiental es un tema multi e interdisciplinario.

\section{Desafíos}

Sin embargo, más allá de los efectos positivos, el desafío es grande y se advierte una necesidad de fortalecer el trabajo en equipo, la colaboración y el diálogo sobre diversos aspectos que dicen relación con la realidad universitaria.

Esto se refleja en las sugerencias de los académicos, tendientes para potenciar la investigación universitaria. Ellos manifiestan:

Yo creo que lo que nos falta en esta Universidad es colocar la realidad muy clara y concreta sobre la mesa... con una enorme transparencia... y poder definir en la Universidad cuáles son las políticas de investigación que va- 
mos a seguir, conocer los niveles de compromiso que hay en esta Universidad en el tema de la investigación.

Pienso que no sería mala idea tener más diálogo sobre la naturaleza propia de la investigación en distintas áreas del conocimiento, que tienen metodologías distintas y propósitos inmediatos distintos, también... Hay investigaciones que son más pragmáticas y otras que son de más largo alcance y que tienen proyecciones en los Proyectos educativos nacionales...

Yo creo que hay un problema de falta de intercambio de experiencias entre la gente que investiga... de ausencia de equipos interdisciplinarios. Esto limita las capacidades individuales para enfrentar correctamente los problemas de investigación... Sería mucho más rica la respuesta, más eficaz, si uno tuviera en cuenta las experiencias de los demás

Yo diría que lo más importante para hacer investigación es tener un enorme grado de humildad... El que crea que es un experto mundial en un tema está perdido... El éxito pasa por aquellos investigadores que están agrupados, que trabajan en conjunto... Esos son los que trabajan más rápido... Además, se deben generar grupos en que se debe reconocer el liderazgo, pero los que lideran deben respetar los espacios para desarrollarse de los demás miembros del grupo y... no utilizar esa capacidad de liderazgo en beneficio propio, porque o si no, el sistema no funciona... ese es el gran desafío.

\section{Conclusiones}

Las percepciones de los investigadores vienen a confirmar, por una parte, la constante interacción entre el interés científico y las posibilidades de acceder a recursos para ejecutar su investigación y, por otra, la necesidad de generar redes científicas con el objeto de potenciar la construcción del conocimiento científico y tecnológico.

Los aspectos antes mencionados constituyen elementos de vital importancia en el quehacer universitario y favorecen la generación de nuevos proyectos, así como la reflexión acerca de la relevancia y vigencia de los temas investigados.

En este contexto, surgen grandes desafíos relacionados con nuevos escenarios de la realidad universitaria que deberemos enfrentar, sin duda, en un futuro cercano. Por cierto, se advierte la necesidad de fortalecer los espacios de comunicación, de diálogo permanente, de trabajo colaborativo en diferentes áreas y temáticas. Se hace urgente y necesario conocer los niveles de compromiso que tienen los académicos con la investigación y discutir sobre naturaleza propia de esta labor, en diferentes áreas del conocimiento. 
Las entrevistas revelaron ciertas condiciones personales que cobran especial relevancia en la generación de las redes de contacto por parte de los investigadores, tales como: capacidad de gestión, apertura, respeto mutuo, disposición para conformar y participar en equipos de trabajo.

Por otra parte, respecto de las posibles proyecciones de este estudio, sería interesante escubrir nuevos aspectos del medio ambiente de investigación en la Universidad y abordar el tema desde una perspectiva fenomenológica. El estudio se podría ampliar, abarcando otras áreas, como el área de educación, el área de la salud, el área de leyes, el área de la creación artística etc.

Cabe señalar que el realizar un estudio comparativo sobre medio ambientes de investigación entre Universidades regionales y extranjeras, constituiría un aporte para el avance de las redes sociales y científicas.

Por otra parte, el investigar lo que ocurre con las redes de conocimiento, de intercambio de información, de colaboración entre compañeros, cooperación inter-escolar o inter-universitaria y su incidencia en los niveles de aprendizaje de los estudiantes, constituiría una ayuda para mejorar procesos pedagógicos, en los casos que así lo requieran.

Recebido em outubro de 2001 e aprovado em fevereiro de 2002.

Referencias bibliograficas

ARANCIBIA, V. Teorías de aprendizaje: revisión de corrientes actuales. Santiago: Cide, 1990.

ASTOLFI, J. P. L'école pour apprendre. Paris: E.S.F., 1992.

AUSUBEL, D.; NOVAK, J.; HANESIAN, H. Psicología educativa. México, DF: Trillas, 1983.

BARRON,A. Constructivismo y desarrollo de aprendizajes significativos. Rev. de Educación, Santiago (Chile), no 294, 301-302, 1991.

BARNES, B. Interest and growth of the knowledge. London: Routledge \& K. Paul, 1981.

BISQUERRA ALZINA, R. Métodos de investigación educativa: guía práctica. Barcelona: CEAC, 1989. 
BRIONES, G. Métodos y técnicas de investigación para las ciencias sociales. México, DF: Trillas, 1990.

CASSIER, M. Le partage des connaissances dans les réseaux scientifiques: l'invention des règles de "bonne conduite" par les chercheurs. Revue Française de Sociologie, Paris, v. 39, no 4, p. 701-720, 1998.

CASTRO HIDALGO, A. Reflexiones acerca de la cognición y tendencia actual de la innovación pedagógica. Paideia: revista de educación, Concepción (Chile), no 26, 1999.

CASTRO HIDALGO, A. La universidad tradicional y la "otra" universidad. 1992. Tese (Doutorado) - Universidad de Uppsala, Suecia, 1992.

COMISIÓN NACIONAL DE INVESTIGACIÓN CIENTÍFICA Y TECNOLÓGICA (CONICYT). Análisis y proyecciones de la ciencia chilena. Santiago: Academia Chilena de Ciencias, 1993.

GUSTAVSSON, S. Debatte om forskningen och sambället. Stockholm: Almquvist \& Wiksell, 1971.

LATOUR, B. Le métier de chercheur, regard d'un antropologue. Paris: INRA, 1995.

MARTON, F. Describing conceptions of the world around us. Göteborg: Institute of Education/ University of Göteborg, 1978. p. 66.

MERTON, R. The sociology of science. Chicago: University Press of Chicago, 1973.

MONTEALEGRE, R. El lenguaje egocéntrico en la psicología contemporánea: polémicas e investigaciones. Revista Latinoamericana de Psicología, Bogotá, v. 26, no 1, p. 11-128, 1994.

NEWMAN, D.; GRIFFIN, P.; COLE, M. La zona de construcción del conocimiento: trabajando por un cambio cognitivo en educación. Madrid: Morata, 1998.

RIVIERE, A. La psicología de Vygotsky. Madrid: Visor, 1988.

SORIA, O. El dilema entre saber, poder y querer: ¿Una nueva universidad para el siglo XXI? Universidades, Tucumán, jul./dic. 1993.

TAYLOR, S.J.; BODGAN, R. Introducción a los métodos cualitativos de investigación: la búsqueda de significados. Buenos Aires: Paidós, 1986. 
UNIVERSIDAD DE CONCEPCIÓN (Chile). Documento de Claustro Académico. Concepción, 1998.

VINCK, Dominique. Sociologie des sciences. Paris: A. Colin, 1995.

WATZLAWICK, P. et al. La realidad inventada. Barcelona: Gedisa, 1990. 